\title{
SPATIALLY RESOLVED CHEMISTRY ON BIMETALLIC SURFACES
}

\author{
R.J. BEHM \\ Abt. Oberflächenchemie und Katalyse, Univ. Ulm, 89069 Ulm, Germany
}

The local chemical properties of bimetallic surfaces, which are often drastically different from those of each of the components, will be discussed. Using $\mathrm{CO}$ adsorption as a probe molecule it will be shown for two model systems, $\mathrm{Au} / \mathrm{Pd}(111)$ and $\mathrm{Pt} / \mathrm{Ru}(0001)$, that their chemical properties depend decisively on the local surface structure and that the correct interpretation of area integrating spectroscopic and kinetic data obtained from such surfaces requires detailed knowledge of their (defect) structure and of the distribution of the different components in the surface layer. It will further be shown that information on the local chemical properties of specific structural elements such as monolayer islands and monolayer island edges, and specific surface ensembles can be gained by applying high resolution scanning tunneling microscopy imaging and area integrating spectroscopic techniques in combination to bimetallic surfaces whose morphology and composition is varied in a systematic and controlled way. Based on experimental results adsorption on a monolayer A / substrate B system is suggested as a model for gaining information on the modifications in chemical properties of $A B$ alloy surfaces due to metal-metal interactions.

PACS numbers: $61.16 . \mathrm{Ch}, 68.10 . \mathrm{Jy}, 82.65 .-\mathrm{i}$

\section{Introduction}

There is a long tradition in the investigation of adsorption and reaction processes on single crystal metal surfaces as a model for heterogeneous catalysis and for catalytic reactions [1]. The effect of promotors was studied by coadsorption of the respective elements, e.g., of alkali metals [2]. The question of catalytically active sites on the small, catalytically active particles was followed up by investigating highly stepped, vicinal surfaces, where the step and kink sites were regarded as potential candidates for these active sites $[3,4]$. In order to model the catalytic properties of bimetallic catalysts, which are well known for their often superior catalytic performance [5], bimetallic surfaces of alloy single crystals or of monometallic substrates (partly) covered by an epitaxial film of a second metal species were investigated (see [6-10] and references therein).

From the modification in the chemical properties, i.e., the changes in the adsorption and reaction behavior imposed by the respective additives or structural elements, these studies gain information on the role of these species in the reaction 
process. Mechanistic information is derived often from kinetic measurements [11]. While deducing the reaction mechanism from reaction kinetics is already problematic and not at all unambiguous on homogeneous surfaces, the situation is even less clear on inhomogeneous surfaces. Though the overall concentration and periodic arrangement of additives, structural elements, etc. can be determined from spectroscopic or diffraction techniques, details on their distribution and local arrangement are largely unknown. For the case of bimetallic surfaces two mechanistic concepts have been introduced to account for the experimentally observed pronounced dependence of the reaction rates on the surface composition, the so-called "ensemble effect" and the "ligand effect" $[12,13]$. The former assumes that a minimum of neighboring surface atoms of one type is required to allow adsorption or reaction on the surface to take place [13], which in particular for surfaces consisting of a disperse mixture of two or more atomic species yields a pronounced concentration dependence. The ligand effect describes the stepwise change in electronic and chemical character of a surface atom $\mathrm{A}$ when the neighboring (surface) atoms are successively replaced by B-type metal atoms [12].

The difficulties in extracting fundamental mechanistic information for reactions on inhomogeneous bimetallic surfaces underline the need for studies on the surface chemistry of well-defined bimetallic surfaces. Further progress in the understanding of the chemistry of these surfaces requires detailed knowledge of the distribution and local arrangement of the individual surface components and structural elements on the one side and the identification of the chemical properties of distinct structural elements and local ensembles on the other side.

The present article is intended to give an overview on the current status of surface chemistry studies on structurally well-defined bimetallic surfaces, which aim at the mechanistic understanding of adsorption and reaction processes on these surfaces on the basis of locally resolved chemical information. This will involve a controlled preparation of bimetallic surfaces with specific local structural elements such as edge atoms, etc. or ensembles of two kinds of metal atoms in specific arrangements ("ensembles"), and the subsequent spectroscopic characterization of the respective adsorption or reaction processes. The most direct characterization of the resulting surfaces will be via STM imaging, which in the case of surface alloy ensembles requires atomic resolution images with chemical contrast in order to distinguish between the different atomic species. We will concentrate here on surfaces prepared by epitaxial growth, by depositing a metal B on a metal A substrate and subsequent surface alloy formation by annealing if required. It should be mentioned that similar studies are of course equally possible on surfaces of bulk alloys [14].

Since these studies depend critically on the controlled preparation of bimetallic surfaces, with a characteristic surface structure, we will first describe basic aspects of metal-on-metal epitaxy relevant for this purpose, including exchange and intermixing processes that are required for alloy and surface alloy formation. The following sections will focus on characteristic aspects of adsorption and reaction processes on two different types of surfaces, first on metal substrates covered by a homogeneous, flat metal (bi-)layer and then on surface alloys with an inhomogeneous surface structure or composition. 


\section{Preparation of bimetallic surfaces by epitaxial growth}

Epitaxial growth has long been described in terms of the three growth modes described by Bauer [15], (continuous) layer-by-layer or Frank-van der Merwe growth, layer-plus-island or Stranski-Krastanov growth and Vollmer-Weber growth, where three-dimensional island growth proceeds from the beginning. It is important to realize, however, that the classification into these growth modes is based on thermodynamic properties such as the surface free energy of substrate and deposit and the interface free energy of the interface between the two, and that under standard deposition conditions metal-on-metal epitaxy is dominated by kinetic limitations such as nucleation, lateral (2D) growth and vertical growth. In this case film growth is determined by nucleation and transport processes, where the latter include intralayer and interlayer transport $[16,17]$. In an atomistic picture these processes are dominated by adatom migration over the free substrate surface (nucleation), migration along island edges (2D growth) and migration across island and step edges (vertical growth). The decisive parameters herefore are the preexponentials and activation energies for the respective migration steps (see $[18,19]$ and references therein).

Considering the present objective of producing bimetallic model catalyst surfaces we have to distinguish between surfaces where a substrate $A$ is covered by a flat, closed layer of a metal deposit and those where either the surface is not fully covered or where, by subsequent annealing or by codeposition of both metals, a surface alloy is prepared. The former, where the surface consists only of one component, would be representative for a cherry structure of the small catalyst particles [20]. The cover layer may be one or more layers thick, but one may expect that with increasing thickness the chemical properties readily relax to those of a B-type bulk metal surface. In the second case the surface contains both components and different bimetallic sites are formed. This would correspond to a fully intermixed particle with both components dispersed on the surface and in the bulk.

We will first focus on the former case of a surface fully covered by a flat, homogeneous metal film (see sketch in Fig. 1c). As can be seen in the next section it may be very important to avoid inhomogeneities such as bilayer islands on a monolayer film, both because of the different chemical properties of a bilayer film as compared to a monolayer film and because of the chemical activity of the island edges introduced by the second layer islands. This also means that the film should be continuous and not exhibit pinholes exposing the bare substrate. In total, a very close coverage control is required. Except for these problems the preparation of continuous, flat monolayer films is rather simple because of the dominating Stranski-Krastanov growth mode in metal-on-metal heteroepitaxy. In this case the deposition or annealing temperature should be high enough to avoid nucleation of next layer islands or remove these before (almost) complete filling of the first layer. On the other hand one has to stay below the onset of surface alloy formation, by exchange and intermixing at the interface. The coverage may be controlled by STM or, if the adsorption energies of the first and second layer are sufficiently different, by thermal desorption spectroscopy [10]. For closed bilayer films the 
a)

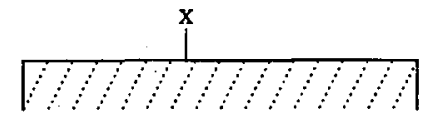

b)

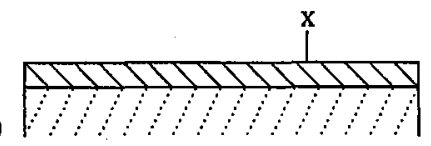

c)

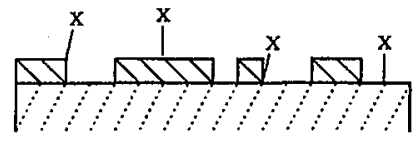

d)

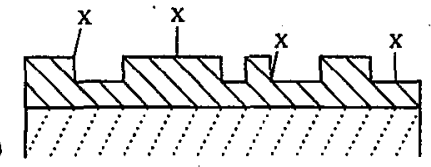

e)

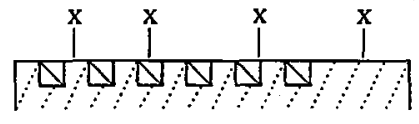

f)

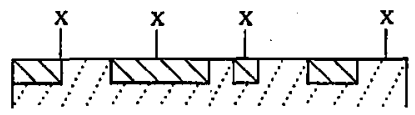

Fig. 1. Sketch indicating different configurations for epitaxially grown bimetallic surfaces and different adsorption sites thereon; (a) monometallic flat surface, (b) surface with monolayer islands on a different metal substrate, (c) surface covered by a flat heteroepitaxial monolayer, (d) as (c) but with second layer islands on top, (e) surface alloy with disperse substitutional atoms $A$ in a surrounding of metal $B$, (f) surface alloy with substitutional monolayer metal B islands in a metal A surface layer.

situation is generally more complicated in that the thermodynamic driving force for this is either small or, if the transition to 3D growth occurs already after completion of the first layer, not existent. In that case the film morphology must be optimized by exploiting the kinetic properties of the growth process, e.g., by a proper choice of the deposition parameters. More complicated procedures involve sequential deposition, either at different temperatures or with an intermediate annealing step, which allows us to modify the size of first layer islands at the onset of second layer nucleation. For instance, in the latter case nucleation of second layer islands will take place on an ideal homogeneous first layer instead of on small first layer islands, avoiding nucleation on individual islands or at first layer island edges.

Recipes for the preparation of inhomogeneous films are much less general and depend strongly on the respective surface aimed for. Mostly it is also not possible to prepare a surface with only a single type of characteristic structural element, and the chemical properties of these sites can only be identified and quantified by varying their concentration over a wide range, possibly without introducing new types of sites.

For characterizing the chemistry of bimetallic sites at the edge of metal B monolayer islands on a metal A substrate (see Fig. 1b) the concentration of these sites can be varied by reducing the deposition temperature. Following the standard nucleation theory lower temperatures lead to a higher density of stable nuclei [16], and hence to smaller island sizes. Maintaining the coverage these sites can be distinguished from adsorption on top of the islands by the increasing fraction of edge atoms with lower deposition temperatures. This effect is illustrated in the 
STM images in Fig. 2, showing two partly Au covered $\mathrm{Pd}(111)$ surfaces after $\mathrm{Au}$ deposition at $100 \mathrm{~K}$ (Fig. 2a, $0.26 \mathrm{ML}$ ) and $300 \mathrm{~K}$ (Fig. 2b, 0.2 ML), respectively. The decrease in temperature led to an increase in the fraction of edge atoms (" $2 \mathrm{D}$ dispersion") from $7 \%$ at $300 \mathrm{~K}$ to $40 \%$ at $100 \mathrm{~K}$ deposition [21]. The resulting changes in the $\mathrm{CO}$ adsorption behavior on these surfaces will be discussed in the last section.
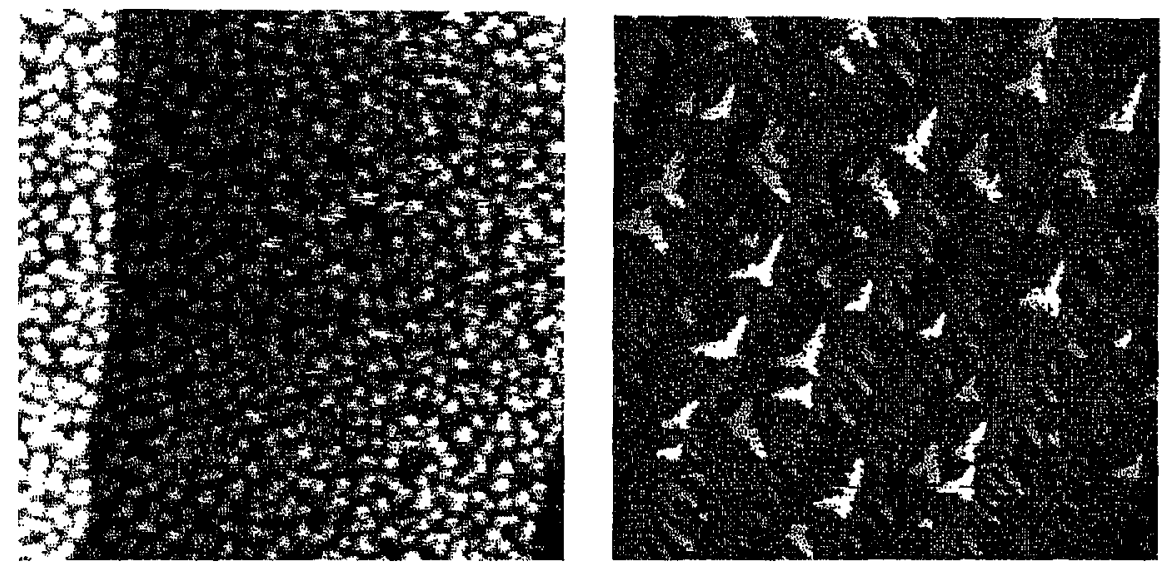

Fig. 2. STM images of a submonolayer $A$ u covered $P d(111)$ surface after deposition of (a) $0.26 \mathrm{ML} \mathrm{Au}$ at $100 \mathrm{~K}$ (flux $\approx 0.8 \mathrm{ML} / \mathrm{min}, 830 \AA \times 830 \AA$ ) and (b) after deposition of $0.2 \mathrm{ML} \mathrm{Au}$ at $300 \mathrm{~K}$ (flux $0.8 \mathrm{ML} / \mathrm{min}, 3300 \AA \times 3300 \AA$ ). In contrast to the small, only slightly ramified islands obtained at $100 \mathrm{~K}$ relaxed dendritic island shapes with preferential growth directions along $\langle 2 \overline{1} \overline{1}\rangle$ develop at $300 \mathrm{~K}[21]$.

Similar procedures may also be applied in order to vary the 2D dispersion of second layer islands (Fig. 1d), which can help, e.g., to discriminate the role of second layer islands and island edges from adsorption or reaction on the first layer. Figure 3 shows STM images for the same system, after deposition of $1.5 \mathrm{ML} \mathrm{Au}$ at $320 \mathrm{~K}$ (Fig. 3a) and after a second deposition of $0.25 \mathrm{ML}$ Au at $110 \mathrm{~K}$ on top of the former surface (Fig. $3 \mathrm{~b}$ ). While $320 \mathrm{~K}$ deposition causes rather large second layer islands to grow on a practically perfect monolayer film, a much larger number of relatively small islands grows after the second Au dose at low temperatures. In this case the fraction of edge atoms is further increased by the ramified shape of the new $\mathrm{Au}$ islands, indicative of very little adatom mobility along the Au island edges. For further details see Ref. [22].

In a large number of metal-on-metal combinations there is a tendency for intermixing, i.e., for the formation of bulk or surface alloys [23, 24]. This process is initiated by the exchange of adatoms with atoms in the surface layer, either upon deposition ("ballistic exchange") [25] or, predominantly, as an activated step, competing with surface migration [26]. In the latter case growth follows the normal "nucleation and growth" pattern at lower temperatures, whereas above a critical temperature adatom-surface exchange sets in, which in addition to creating mixed 

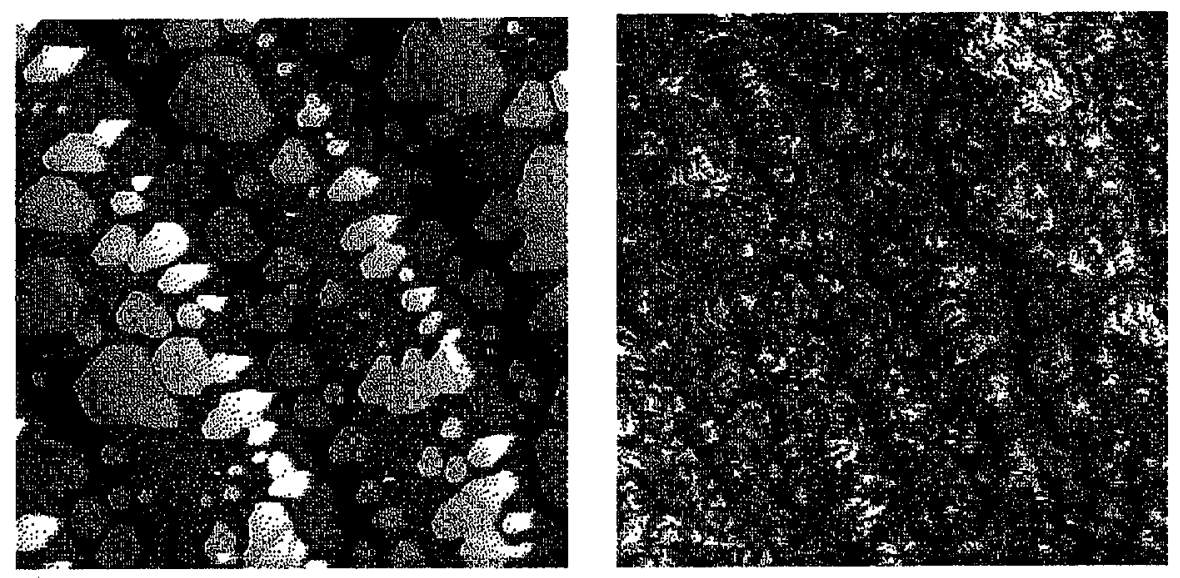

Fig. 3. STM images of a $\operatorname{Pd}(111)$ surface covered (a) by $1.5 \mathrm{ML} \mathrm{Au}\left(T_{\text {dep }}=320 \mathrm{~K}\right.$; $3350 \AA \times 3350 \AA$ ) and (b) after a second deposition of $0.25 \mathrm{ML}$ Au on the surface shown in (a) at $110 \mathrm{~K}(3450 \AA \times 3450 \AA)[22]$.

surface layers may affect the growth behavior in various ways [26-28]. The critical temperature depends on the activation barrier for exchange and reaches from far below room temperature, as observed, e.g., for $\mathrm{Ni} / \mathrm{Ag}(100)$ [29] or $\mathrm{Rh} / \mathrm{Ag}(100)$ [28], up to temperatures well above $1000 \mathrm{~K}$, as found for $\mathrm{Pt} / \mathrm{Ru}(0001)$ [30]. Furthermore, it had been shown that specific sites on the surface such as deposit island edges or substrate steps may be preferred sites for exchange to occur [31]. Hence, similar to standard growth also here both thermodynamic and kinetic effects have to be considered. Finally it should be noted that these exchange and intermixing processes not only occur during deposition, but also upon annealing a metal film covered surface. In general, the onset of intermixing in this case is at higher temperatures than during deposition, because of the mutual stabilization of the metal atoms in a closed layer.

The most important structural aspect in these bimetallic surface layers for the current purpose is the concentration and distribution of the respective atoms. Ion scattering spectroscopy (ISS) gives a very precise determination of the concentration in the topmost layer, while electron spectroscopies such as Auger electron spectroscopy (AES) and X-ray photoelectron spectroscopy (XPS) also contain contributions from deeper layers. More directly, the concentration in the surface layer can be determined from atomic resolution STM images with chemical contrast. As shown in the STM image in Fig. 4 recorded on a $\mathrm{Pt}$ containing $\mathrm{Ru}(0001)$ surface [32], the different atomic species can clearly be differentiated. After deposition of $0.42 \mathrm{ML} \mathrm{Pt}$ at $300 \mathrm{~K}$ and subsequent annealing to $1200 \mathrm{~K}$ the surface contains about $31 \% \mathrm{Pt}$ atoms, which are almost statistically distributed over the surface (the assignment of "dark" and "bright" atoms to Pt and Ru was made on the basis of parallel AES measurements [30]). The quantitative evaluation of such images allows a direct and unambiguous determination not only of surface concentrations, but also of correlations in the surface atom distribution. For the surface 


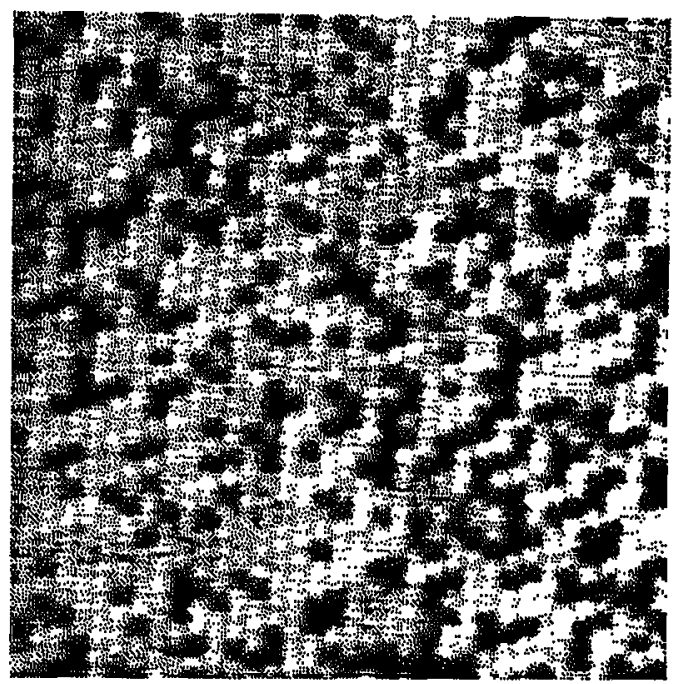

Fig. 4. Atomic resolution image of a $\mathrm{Pt} / \mathrm{Ru}(0001)$ obtained by deposition of $0.42 \mathrm{ML}$ $\mathrm{Pt}$ at $300 \mathrm{~K}$ and subsequent annealing to $1200 \mathrm{~K}(85 \AA \times 102 \AA)$. Pt atoms show up as "black" lower lying atoms in this image [32].

shown in Fig. 4 this showed that the substitutional Pt atoms are slightly more aggregated than expected for a statistical arrangement. In addition to containing information on the interactions between the respective atoms this information is a basic requirement for the proof and quantification of ensemble effects etc. Hence knowledge of the atom distribution is the basis for a detailed understanding of adsorption and reaction processes on these surfaces, and also for theoretical work on this topic.

\section{Surface chemistry on homogeneous bimetallic surfaces}

Homogeneous bimetallic surfaces, with a metal B film covering a metal A substrate, differ from the clean substrate B by the different geometric and electronic structure of the substrate. The former may lead to the formation of strained lattices, where the adatom-adatom spacing differs from that on the respective bulk metal surface. It was shown in both experimental and theoretical studies that this effect can severely affect the barrier for metal adatom migration, and similar effects are also expected for the adsorption of molecules on top of these deposit layers [33, 34]. These effects will increase with strain, the strongest effects will therefore be expected for a large lattice mismatch in combination with pseudomorphic growth, i.e., with no strain relaxation. Second, the electronic structure of the metal deposit may be modified by coupling to the chemically different substrate, which is reflected, e.g., by the formation of specific interface states $[10,35,36]$. These effects are discussed in detail and reviewed for a large number of systems in Ref. [10]. It is very important to realize that the surface atoms in a metal B monoor bilayer film on a different metal substrate are chemically no longer identical to a metal B surface, but may differ markedly. 




Fig. 5. Set of TD spectra obtained after saturation (10 L exposure) of (a) clean $\mathrm{Ru}(0001)$, (b) $\mathrm{Ru}(0001)$ with $1.1 \mathrm{ML} \mathrm{Pt}$ deposited at $300 \mathrm{~K}$ and annealed to $800 \mathrm{~K}$, (c) $\mathrm{Ru}(0001)$ with $2.5 \mathrm{ML} \mathrm{Pt}$ deposited at $300 \mathrm{~K}$ and annealed to $800 \mathrm{~K}$, (d) a $\mathrm{Pt} / \mathrm{Ru}(0001)$ surface alloy with $0.42 \mathrm{ML} \mathrm{Pt}$ deposited at $300 \mathrm{~K}$ and annealed to $1200 \mathrm{~K}$ and (e) a thick Pt film (4 ML) deposited at $300 \mathrm{~K}$.

An example for such modifications is presented in the thermal desorption (TD) spectra in Fig. 5, taken from Ref. [37]. These were obtained for CO saturation adsorption on a pure $\mathrm{Ru}(0001)$ surface (spectrum $a$ ), on the same surface covered by slightly more than $\mathrm{Pt}$ monolayer (1.1 ML) (spectrum $b$ ), on a Ru(0001) surface covered with 2.5 ML Pt (spectrum $c$ ) and on a surface covered with a thick Pt film (4 ML) which should be very similar to that from a Pt(111) surface (spectrum $e$ ). After deposition at $300 \mathrm{~K}$ the $1.1 \mathrm{ML}$ and $2.5 \mathrm{ML}$ Pt films were annealed to $800 \mathrm{~K}$ in order to obtain a flat morphology. For the monolayer film STM images revealed a continuous closed film with a few second layer islands [30]. The 2.5 ML film is slightly rougher. In addition to flat bilayer and trilayer areas also fourth layer islands exist. CO desorption from a saturated $\mathrm{Ru}(0001)$ surface $\left(T_{\mathrm{ad}}=90 \mathrm{~K}, 10 \mathrm{~L}\right)$ results in the well-known double peak spectrum characteristic of this system, with maxima at around $400 \mathrm{~K}$ and $455 \mathrm{~K}[38,39]$. Dramatic changes are observed for going to desorption from a $\mathrm{CO}$ saturated, monolayer $\mathrm{Pt}$ covered surface. This spectrum is shifted by about $100-150 \mathrm{~K}$ to lower temperatures, with the onset of desorption at about $200 \mathrm{~K}$ instead of $300 \mathrm{~K}$, and the high temperature end at about $350 \mathrm{~K}(520 \mathrm{~K}$ for bare $\mathrm{Ru}(0001))$. The small peak centered at around 
$400 \mathrm{~K}$ reflects the presence of residual defects and bilayer islands. The spectrum obtained from the $2.5 \mathrm{ML}$ film is backshifted to higher temperatures. It can be considered as being composed of contributions from two, three and four layer $\mathrm{Pt}$ areas. For the present discussion its most important feature is the fact that the onset of desorption is shifted by about $30 \mathrm{~K}$ to higher temperatures as compared to the monolayer film, indicating that (at saturation coverage) the $\mathrm{CO}$ adsorption energy on a bilayer film is significantly stronger than on a monolayer film. Finally, for CO desorption from the $4 \mathrm{ML}$ Pt film the spectrum very much resembles that for $\mathrm{CO}$ desorption from a $\mathrm{Pt}(111)$ surface [40]. The onset of desorption is shifted by another $50 \mathrm{~K}$ to higher temperatures, reflecting a further increase in CO adsorption energy. The shift in desorption temperature on the mono- and bilayer $\mathrm{Pt}$ covered $\mathrm{Ru}(0001)$ surface as compared to desorption from bulk $\mathrm{Pt}(111)$ resembles that observed for $\mathrm{Pd} / \mathrm{Ru}(0001)$, but is even more pronounced [41]. Along with these modifications in the adsorption properties of the Pd surface layer the authors also reported a significant shift in the $\mathrm{Pd}(3 d)$ core level energies as compared to bulk $\mathrm{Pd}$, indicative of changes in the electronic structure of the Pd layer. Further details on the $\mathrm{CO}$ adsorption and oxidation behavior on bimetallic $\mathrm{Pt}-\mathrm{Ru}(0001)$ surfaces will be published elsewhere [32, 37].

In conclusion, while CO is strongly adsorbed on both Pt(111) and $\mathrm{Ru}(0001)$, it is much weaker absorbed on a Pt modified $\mathrm{Ru}(0001)$ surface covered with a $\mathrm{Pt}$ mono- or bilayer, respectively. The strongest reduction in adsorption energy occurs on the monolayer covered surface. The implications for catalytic purposes become evident by comparison with previous discussion on the catalytic activity of PtRu catalysts in polymer electrolyte fuel cells ("bifunctional catalysis") [42, 43]. At the usual operation temperatures these catalysts suffer from poisoning due to $\mathrm{CO}$ adsorption, which is present in trace amounts in the fuel gas in $\mathrm{H}_{2}$ operated cells or results as a side product in the direct oxidation of methanol in methanol operated cells $[44,45]$. The quality of a catalyst for low temperature fuel cell applications therefore depends critically on its $\mathrm{CO}$ tolerance [46]. The increased $\mathrm{CO}$ tolerance of $\mathrm{PtRu}$ catalysts as compared to pure $\mathrm{Pt}$ or Ru catalysts [47] can be explained by the change in the adsorption properties of modified $\mathrm{Pt}$, which reduces the adsorption energy and, due to the strongly enhanced desorption rate, also the steady state concentration of adsorbed $\mathrm{CO}$ drastically. As will be shown in the next section, qualitatively similar results are obtained on PtRu surface alloys.

\section{Surface chemistry on surface alloys and other inhomogeneous bimetallic surfaces}

The problems associated with the interpretation of spectroscopic or kinetic data obtained on inhomogeneous surfaces is evident already from the discussion of the CO desorption spectrum from the 2.5 ML Pt covered surface. The clear separation of the individual contributions, of desorption from two-, three- or four-layer $\mathrm{Pt}$ areas would require to vary the film thickness and record similar spectra from surfaces with differing amounts of these areas. In other cases adsorbates on specific surface sites can be identified by spectroscopic means. This will be demonstrated in the following example, dealing with $\mathrm{CO}$ adsorption on bimetallic $\mathrm{Au}-\mathrm{Pd}(111)$ surfaces (further details see in Refs. [21, 22, 48]). 
For $\mathrm{Pd}(111)$ covered by submonolayer amounts of $\mathrm{Au}$ (deposition temperature $300 \mathrm{~K}$ ) it was shown that upon $\mathrm{CO}$ adsorption a small extra peak at $263 \mathrm{meV}$ appears in HREEL spectra, in addition to the main C-O stretch peak at $245 \mathrm{meV}$ associated with $\mathrm{CO}$ adsorbed on the $\mathrm{Pd}(111)$ surface areas [21]. While the presence of this extra state is clearly connected with the Au deposit, the question of the adsorption site of these $\mathrm{CO}$ molecules, e.g., on top of the islands or at the edges, cannot be answered from these data. This can be decided by varying the deposition temperature. By going from $300 \mathrm{~K}$ to $100 \mathrm{~K}$, at a coverage of about $0.2 \mathrm{ML}$, the surface changes from the configuration shown in Fig. $2 \mathrm{a}$ to that in Fig. 2b, with a much higher density of islands and, important in this context, of island edges after low temperature deposition. The 2D dispersion was mentioned before to increase from about $7 \%$ upon deposition at $300 \mathrm{~K}$ to about $40 \%$ upon deposition at $100 \mathrm{~K}$. The HREEL spectra recorded after $0.5 \mathrm{~L}$ CO exposure to these two surfaces $\left(T_{\mathrm{ad}}=100 \mathrm{~K}\right.$ ) are shown in Fig. 6 (spectra $b$ and $c$ ), together with a spectrum recorded on the pure $\operatorname{Pd}(111)$ surface after similar exposure (spectrum a). The pronounced increase in the $263 \mathrm{meV}$ loss feature in spectrum $c$ provides clear evidence that this extra state is associated with $\mathrm{CO}$ adsorbed at the $\mathrm{Au}$ island edges. Furthermore, adsorption on the islands can be excluded also from the experimental finding that a $\mathrm{Pd}(111)$ surface covered by a full monolayer of $\mathrm{Au}$ is practically inert against $\mathrm{CO}$ adsorption at $100 \mathrm{~K}$ [21].

The next question relates to the adsorption site of the new $\mathrm{CO}$ species. The high frequency of $263 \mathrm{meV}$ for the $\mathrm{C}-\mathrm{O}$ stretch vibration points to a linear $\mathrm{CO}$ species adsorbed in an on-top configuration. This leaves the possibility of $\mathrm{CO}$ adsorption on a modified $\mathrm{Pd}$ atom at the lower side of the step or on a modified $\mathrm{Au}$ atom at the upper side of the island edge (see Figs. 1b, c). A recent comparison between $\mathrm{Pd}(111)$ surfaces covered by a full Au monolayer with different densities of second layer $\mathrm{Au}$ islands on top - the morphology of these surfaces is shown in Figs. 3a and $\mathrm{b}$ - found a similar $\mathrm{CO}$ state also for second layer Au islands and no adsorption on the flat bilayer areas, and the same observations were made also on a thicker Au film [22]. These results provide clear evidence that the extra state is due to $\mathrm{CO}$ adsorption on top of the $\mathrm{Au}$ island edge atoms, and not at $\mathrm{Pd}$ atoms (submonolayer coverages) or Au atoms (above monolayer coverages) at the lower side of the edge. Hence, the data point to a significant stabilization of the $\mathrm{Au}-\mathrm{CO}$ bond for $\mathrm{CO}$ adsorbed on Au edge atoms. This conclusion agrees perfectly well also with recent theoretical results on the stabilization of $\mathrm{CO}$ on stepped $\mathrm{Pt}$ surfaces [49]. Those authors found a significant stabilization for $\mathrm{CO}$ adsorbed on $\mathrm{Pt}$ edge atoms by about $0.7 \mathrm{eV}$ as compared to adsorption on $\mathrm{Pt}(111)$ terrace sites, which they explained by the better coupling of the $2 \pi^{*} \mathrm{CO}$ orbital with the $\mathrm{Pt} d$-electrons due to an upshift of the $d$-band towards the Fermi level at the step sites. While these calculations were specifically performed for $\mathrm{CO}$ adsorption on $\mathrm{Pt}$ surfaces, the basic conclusion of a more stable $\mathrm{CO}$ bond to undercoordinated edge atoms is correct also for other metal surfaces [50].

The result of a stabilization of $\mathrm{CO}$ adsorbed on edge atoms should hold true of course also for the submonolayer and higher coverage $\mathrm{Pt}$ films on $\mathrm{Ru}(0001)$ discussed in more detail in the last section. In all cases the TD spectra exhibited a more or less pronounced additional desorption peak at higher temperatures, 


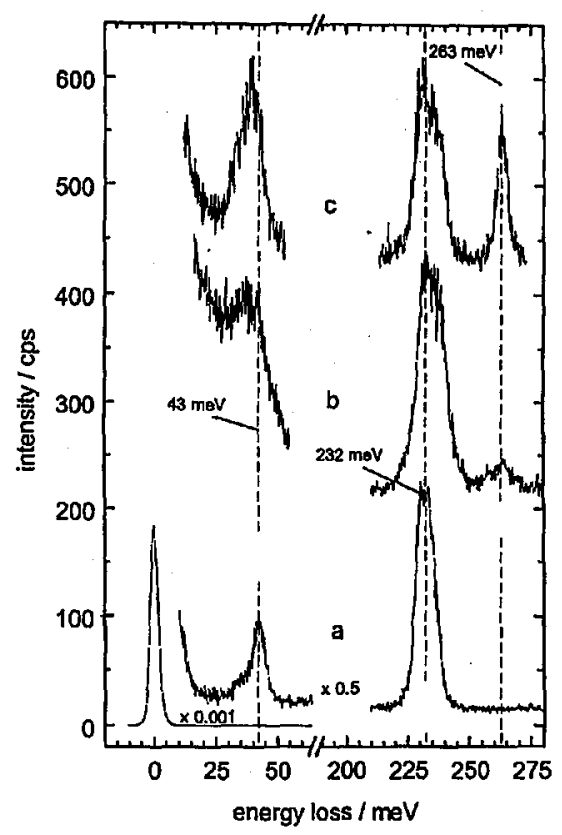

Fig. 6. Set of HREEL spectra obtained after $0.5 \mathrm{~L}$ CO adsorption on a clean Pd(111) surface (a) and bimetallic $\mathrm{Au} / \mathrm{Pd}(111)$ surfaces $\left(T_{\mathrm{ads}}=100 \mathrm{~K}, p_{\mathrm{ads}}=10^{-8} \mathrm{mbar}\right)$ prepared by $0.2 \mathrm{ML}$ Au deposition at $300 \mathrm{~K}(\mathrm{~b})$ and $0.2 \mathrm{ML}$ Au deposition at $110 \mathrm{~K}$ (c). The spectra are normalized to equal intensity in the elastic peak [21].

depending on the respective preparation procedure. The higher adsorption energy reflected by this peak is compatible with the above explanation, although for medium $\mathrm{Pt}$ coverages desorption from areas with a locally higher thickness (bilayer islands in a monolayer film, trilayer areas on a bilayer film) lead to similar results.

As a second point, adsorption and reaction on bimetallic (surface) alloys with both components in the surface layer shall be discussed. Based on the previous results and discussion one would expect that the mutual interactions between the different atomic species in the surface lead to a modification in their electronic and chemical properties, similar to the case of the monolayer film on a different substrate. These modifications should be most pronounced for the case of individual, disperse atoms in a surrounding of the other component (Fig. 1e), while small $2 \mathrm{D}$ ensembles of the one metal in a matrix of the other metal (Fig. If) come close to the situation of the monolayer island (Fig. 1b), except for the absence of the island edges. Finally, coexisting 3D clusters should tend in their chemical properties towards the respective bulk metals surfaces, again not considering the transition regions between the two.

This picture very much resembles that underlying the "ligand effect", in that the substitution of neighboring (surface) atoms will modify the chemical and electronic properties of the respective central atom being considered. In this picture the monolayer situation is characterized by six neighboring atoms of the same 
kind and three underlying atoms of the other kind. Additional effects due to lattice strain, longer range or higher order interactions are not included. Following that description, surfaces with random mixtures of the individual atoms would in average be somewhere between the two cases of disperse atoms and monolayer islands in their chemical properties.

These ideas can be tested for $\mathrm{CO}$ adsorption on a PtRu surface alloy, with Ru surface atoms in a $\mathrm{Ru}(0001)$ surface substituted by $\mathrm{Pt}$ atoms, and comparing the resulting $\mathrm{CO}$ adsorption behavior with that observed on a $\mathrm{Pt}$ monolayer covered $\mathrm{Ru}(0001)$ surface (see last section). A TD spectrum recorded after $\mathrm{CO}$ saturation of a PtRu surface alloy with $31 \% \mathrm{Pt}$ surface atoms, which was prepared by depositing $0.42 \mathrm{ML} \mathrm{Pt}$ at room temperature and subsequent annealing to $1200 \mathrm{~K}$, is shown as spectrum $d$ in Fig. 5. This spectrum is not very different from spectra obtained from partially $\mathrm{Pt}$ covered samples [32]. The onset of desorption at about $200 \mathrm{~K}$ is identical to that observed on the monolayer film. The desorption range up to $500 \mathrm{~K}$, on the other hand, comes close to that found on pure $\mathrm{Ru}(0001)$ surfaces, as evidenced by comparison with spectrum $a$. One might argue that the close similarity between these spectra is again due to the formation of $\mathrm{Pt}$ islands, but in the surface layer. The high resolution STM image of this surface in Fig. 4, however, provides clear evidence that this assumption is not correct. As mentioned in Sec. 2 the $\mathrm{Pt}$ atoms are dispersely distributed within the topmost layer, with only a slight correlation between the substitutional $\mathrm{Pt}$ atoms [30]. The structure of the surface layer is identical to that of a pure $\mathrm{Ru}(0001)$ surface, and hence identical also to that of the pseudomorphic Pt islands. In a first semiquantitative approach it can be concluded that adsorption on $\mathrm{Pt}$ atoms in the Ru surface layer is of about similar strength as on top of a $\mathrm{Pt}$ monolayer. Since for these Pt concentrations also small $\mathrm{Ru}$ areas exist on the surface, additional experiments with a higher density of $\mathrm{Pt}$ surface atoms are required to gain information on whether and by how much $\mathrm{Ru}$ atoms are modified by neighboring $\mathrm{Pt}$ atoms with respect to $\mathrm{CO}$ adsorption. One should keep in mind also that in these cases the Pt or Ru surface atoms are always adsorbed on a $\mathrm{Ru}(0001)$ substrate. Hence modifications of the Pt surface atoms as compared to bulk Pt are more plausible than for Ru surface atoms. In a bulk alloy the situation would be different in that also Ru surface atoms might equally well also reside on a (local) Pt underlayer. This situation could be modeled by adsorption measurements on a Ru monolayer covered $\mathrm{Pt}(111)$ substrate.

\section{Conclusions}

Using $\mathrm{CO}$ adsorption as a probe molecule it was shown for two model systems, $\mathrm{Au} / \mathrm{Pd}(111)$ and $\mathrm{PtRu}(0001)$, that the chemical properties of bimetallic surfaces, which may be drastically different from those of each of the two components, depend decisively on the local surface structure. The correct interpretation of area integrating spectroscopic and kinetic data obtained from such surfaces requires detailed knowledge of their (defect) structure and their surface composition, where the latter includes also the distribution of the different components in the surface layer.

The data show that information on the local chemical properties of specific structural elements such as monolayer islands and monolayer island edges, and 
specific surface ensembles can be gained by combining high resolution STM imaging with area integrating spectroscopic techniques and by systematic, controlled variation of the surface morphology and composition. They also underline the role and importance of structural defects such as steps and island edges in model studies on bimetallic surfaces prepared by metal-on-metal epitaxy.

From the close similarity in trends for $\mathrm{CO}$ adsorption on Pt monolayer covered $\mathrm{Ru}(0001)$ and $\mathrm{PtRu}(0001)$ surface alloys adsorption on a monolayer film of the one component on a substrate of the other component was suggested as a means for gaining qualitative information on the chemical properties of (surface) alloy surfaces composed of these two components. Further. work on these problems is in progress.

\section{Acknowledgments}

I gratefully acknowledge the excellent work by F. Buatier de Mongeot, S. Frey, B. Gleich, M. Ruff, and M. Scherer, which laid the basis for this article, and interesting discussions with H.A. Gasteiger and J.K. Nørskov (Techn. Univ. Denmark).

\section{References}

[1] G.A. Somorjai, Introduction to Surface Chemistry and Catalysis, Wiley, New York 1994.

[2] See in: Physics and Chemistry of Alkali Metal Adsorption, Eds. H.P. Bonzel, A.M. Bradshaw, G. Ertl, in series Materials Science Monographs, Vol. 57, Elsevier, Amsterdam 1989.

[3] S.M. Davies, F. Zaera, G.A. Somorjai, J. Am Chem. Soc. 104, 7453 (1982).

[4] S.M. Davies, F. Zaera, G.A. Somorjai, J. Catal. 85, 296 (1984).

[5] J.H. Sinfelt, Bimetallic Catalysts: Discoveries, Concepts and Applications, Wiley, New York 1983.

[6] C.T. Campbell, Annu. Rev. Phys. Chem. 41, 775 (1990).

[7] B.E. Nieuwenhuys, in: The Chemical Physics of Solid Surfaces, Eds. D.A. King, W.P. Woodruff, Vol. 6, Elsevier, Amsterdam 1993, p. 185.

[8] W.K. Kuhn, R.A. Campbell, D.W. Goodman, in: The Chemical Physics of Solid Surfaces, Eds. D.A. King, W.P. Woodruff, Vol. 6, Elsevier, Amsterdam 1993, p. 157.

[9] B.E. Nieuwenhuys, Surf. Rev. Lett. 3, 1869 (1996).

[10] J.A. Rodriguez, Surf. Sci. Rep. 24, 223 (1996).

[11] J.A. Rodriguez, D.W. Goodman, Surf. Sci. Rep. 1/2, 1 (1991).

[12] Y.L. Lam, J. Criado, M. Boudart, Nouv. J. Chim. 1, 461 (1977).

[13] J.W.A. Sachtler, G.A. Somorjai, J. Catal. 81, 77 (1983).

[14] A. Pantförder, J. Skonieczny, E. Janssen, G. Meister, A. Goldmann, P. Varga, Surf. Sci. 337, 177 (1995).

[15] E. Bauer, Z. Kristallogr. 110, 372 (1958).

[16] J.A. Venables, G.D.T. Spiller, M. Hanbücken, Rep. Prog. Phys. 47, 399 (1974).

[17] S.L. Chang, P.A. Thiel, Crit. Rev. Surf. Chem. 3, 239 (1994).

[18] J.A. Meyer, H.A. van der Vegt, J. Vrijmoeth, E. Vlieg, R.J. Behm, Phys. Rev. B 51,14790 (1995). 
[19] See contributions in: The Chemical Physics of Solid Surfaces, Eds. D.A. King, D.P. Woodruff, Vol. 8, Elsevier, Amsterdam 1997.

[20] W.M.H. Sachtler, R.A. van Santen, Surf. Sci. 3, 121 (1979).

[21] B. Gleich, M. Ruff, R.J. Behm, Surf. Sci. 386, 48 (1997).

[22] M. Ruff, S. Frey, B. Gleich, R.J. Behm, submitted to publication.

[23] A. Christensen, A.V. Ruban, P. Stoltze, K.W. Jacobsen, H.L. Skriver, J.K. Nørskov, F. Besenbacher, in press.

[24] C. Engdahl, P. Stoltze, K.W. Jacobsen, J.K. Nørskov, H.L. Skriver, J. Vac. Sci. Technol. A 12, 1787 (1994).

[25] G.L. Kellogg, Phys. Rev. Lett. 76, 98 (1996).

[26] J.A. Meyer, R.J. Behm, Surf. Sci. 302, L275 (1995).

[27] J.A. Meyer, I.D. Baikie, E. Kopatzki, R.J. Behm, Surf. Sci. 365, L647 (1996).

[28] S.L. Chang, J.-M. Wen, P.A. Thiel, S. Günther, J.A. Meyer, R.J. Behm, Phys. Rev. B 53, 13747 (1996).

[29] R. Dorsch, Ph.D. Thesis, Univ. Ulm, Ulm 1996.

[30] M. Scherer, F. Buatier de Mongeot, R.J. Behm, to be published; M. Scherer, Ph.D. Thesis, Univ. Ulm, Ulm 1996.

[31] M.B. Hugenschmidt, A. Hitzke, R.J. Behm, Phys. Rev. Lett. 76, 2535 (1996).

[32] F. Buatier de Mongeot, B. Gleich, M. Scherer, R.J. Behm, submitted to publication.

[33] H. Brune, K. Bromann, H. Röder, K. Kern, J. Jacobsen, P. Stoltze, K. Jacobsen, J.K. Nørskov, Phys. Rev. B 52, 14380 (1995).

[34] J.A. Meyer, P. Schmid, R.J. Behm, Phys. Rev. Lett. 74, 3864 (1995).

[35] J.E. Houston, J.M. White, P.J. Feibelman, D.R. Haman, Phys. Rev. B 38, 12164 (1986).

[36] J.E. Houston, C.H.F. Peden, P.J. Feibelman, D.R. Haman, Phys. Rev. Lett. 56, 375 (1988).

[37] B. Gleich, M. Buatier de Mongeot, R.J. Behm, to be published.

[38] H. Pfnür, P. Feulner, D. Menzel, J. Chem. Phys. 79, 4613 (1983).

[39] K.L. Kostov, H. Rauscher, D. Menzel, Surf. Sci. 278, 62 (1992).

[40] H. Steininger, S. Lehwald, H. Ibach, Surf. Sci. 123, 264 (1982).

[41] R.A. Campbell, J.A. Rodriguez, D.W. Goodman, Phys. Rev. B 46, 7077 (1992).

[42] M. Watanabe, S. Motoo, J. Electroanal. Chem. 60, 267 (1975); ibid. p. 275.

[43] H.J. Oetjen, V.M. Schmidt, U. Stimming, F. Trila, J. Electrochem. Soc. 143, 3838 (1996).

[44] M.P. Hogarth, G.A. Hards, Platinum Metals Rev. 40, 150 (1996).

[45] T.R. Ralph, Platinum Metals Rev. 41, 102 (1997).

[46] R.A. Lemon, J. Power Sources 29, 251 (1990).

[47] H.A. Gasteiger, N.M. Markovic, P.N. Ross, J. Phys. Chem. 99, 16757 (1995).

[48] M. Ruff, S. Frey, B. Gleich, R.J. Behm, to be published.

[49] B. Hammer, O.H. Nielsen, J.K. Nørskov, Catal. Lett. 46, 31 (1997).

[50] J.K. Nørskov, private communication. 